\title{
Die Zielstrebige
}

\section{BRITTA-JULIA KREUZER Stuttgart - Hildesheim - Südafrika - Bodensee.}

Das sind die Stationen, die Britta-Julia Kreuzer in ihren fünf Jahren also Physiotherapeutin bereits passiert hat. Neben der Physiotherapie befasst sie sich vor allem mit Mitarbeiterführung und Leitungsaufgaben. Fazit ihrer Bachelorarbeit: Fachliche Kompetenz alleine reicht nicht für eine leitende Position.

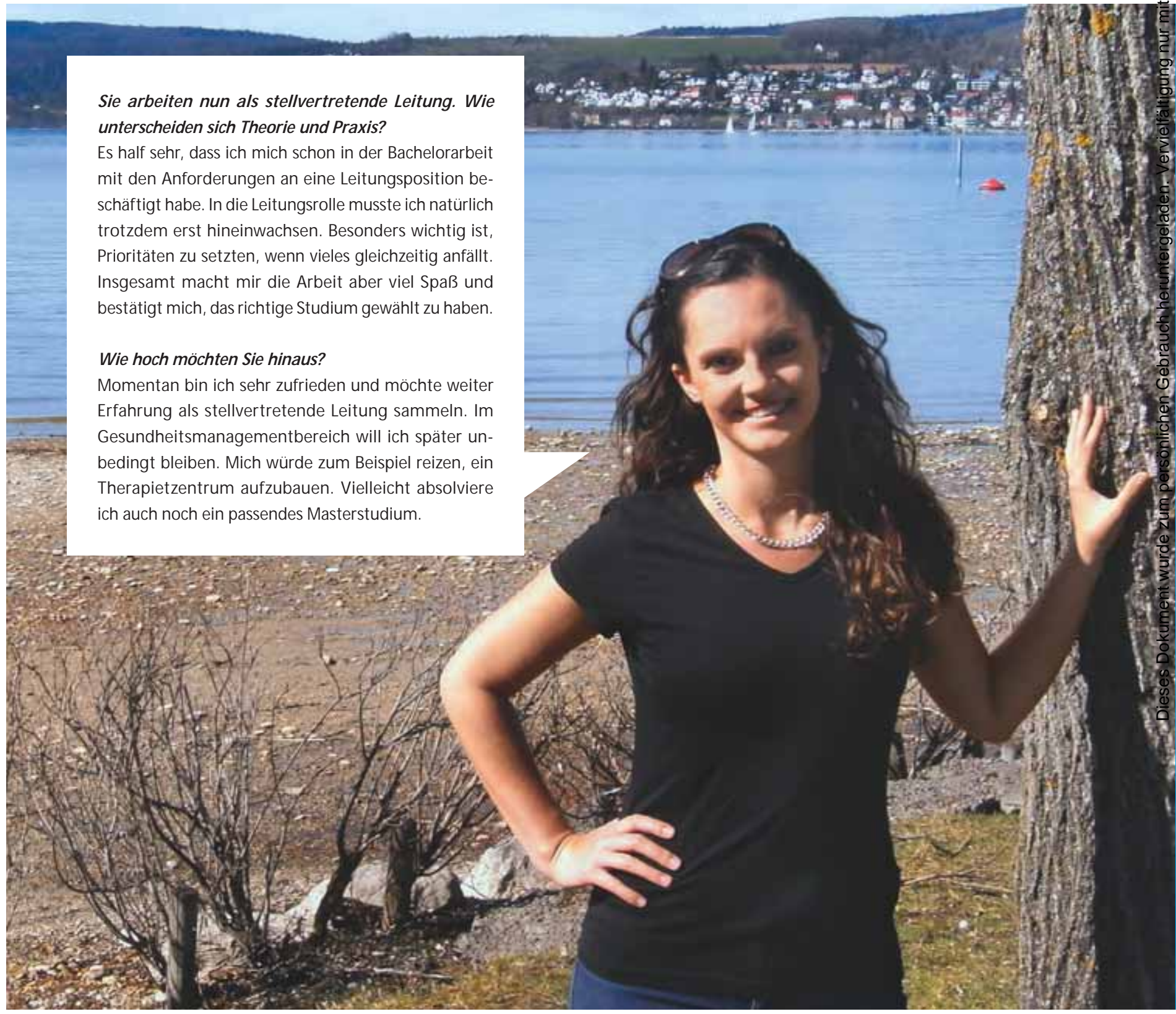




\section{Ansprüche an leitende Therapeuten}

Britta-Julla Kreuzer ...

... ist 28 Jahre alt und lebt und arbeitet in der Schweiz. Im Kanton Thurgau ist sie stellvertretende Therapieleitung in einer Rehabilitationsklinik mit Schwerpunkt Orthopädie. 2008 schloss sie ihre Ausbildung zur Physiotherapeutin in Stuttgart ab. Um unter anderem ihren wissenschaftlichen Background zu vertiefen und einfacher im Ausland arbeiten zu können, machte sie im Anschluss den Bachelorstudiengang an der Hochschule Hildesheim. Über ein Stipendium bekam sie dann die Möglichkeit, ein halbes Jahr in einer Klinik in Kapstadt/Südafrika zu arbeiten, wo sie unter anderem ein Praktikum bei der therapeutischen Leitung absolvieren konnte. Zurück in Deutschland, zog es sie an den schönen Bodensee. Dort arbeitete sie zunächst in einer Rehaklinik. Danach zog sie ans Schweizer Bodenseeufer nach Kreuzlingen. Mit dem Wechsel auf die andere Seite der Grenze wechselte sie auch die Arbeitsstätte und stieg zur stellvertretenden Therapieleitung auf. Sie behandelt weiterhin Patienten, ist aber auch für Leitungsaufgaben eingeplant. In ihrer Freizeit treibt sie gerne Sport, vor allem Tennis, trifft sich mit Freunden und geht auf Reisen. Gerade ist sie von einem weiteren Besuch in Südafrika zurück.

\section{Die Bachelorarbeit}

Motivierte und zufriedene Mitarbeiter sind eine bedeutsame Ressource physiotherapeutischer Einrichtungen. Deshalb sollten für leitende Physiotherapeuten Personalmanagement und insbesondere Personalführung einen großen Stellenwert haben. In vielen Einrichtungen steigen jedoch die dienstältesten oder fachlich kompetentesten Physiotherapeuten in die Führungsebene auf, ohne vorher eine umfassende Qualifizierung erhalten zu haben, die sie auf die vielen neuen Aufgaben vorbereitet.

In ihrer Bachelorarbeit wollte Britta-Julia Kreuzer herausfinden, welche Anforderungen speziell an physiotherapeutische Leitungen in stationären Abteilungen gestellt werden und welche Kompetenzen diese benötigen. Ihre systematische Recherche zeigte, dass es kaum Literatur zum Personalmanagement in der stationären Physiotherapie gibt. Deshalb befasste sich die Physiotherapeutin zunächst allgemein mit den Grundlagen der Mitarbeiterführung. Um das Aufgabenspektrum einer physiotherapeutischen Leitung beschreiben zu können, analysierte sie zudem die Stellenbeschreibungen von drei Therapeuten in leitender Position. Daraus schloss sie die erforderlichen Kompetenzen. Abschließend stellte sie Beispiele motivierender Mitarbeiterführung vor und beschrieb, wie diese in der stationären Physiotherapie umgesetzt werden können.

\section{Ergebnisse}

Britta-Julia Kreuzer hat herausgefunden, dass...

> sich die Leitung meistens auf der mittleren Managementebene in einer sog. SandwichPosition befindet - sie muss also Weisungen der Krankenhausdirektion folgen und gleichzeitig die Bedürfnisse des Teams berücksichtigen.
> die Aufgaben sehr umfangreich sind: Es fallen betriebsbezogene Aufgaben wie Budgetverwaltung und Qualitätsmanagement an sowie personalbezogene Aufgaben, etwa Personalplanung und -entwicklung. Zudem behandelt die Leitung oft noch Patienten.

$>$ die motivierende Personalführung eine zentrale Führungsaufgabe ist. Umsetzungsbeispiele sind Jobrotation - also der freiwillige Wechsel zwischen Stationen mit verschiedenen Schwerpunkten (z. B. Gynäkologie Orthopädie - Chirurgie) - , kollegiale Qualitätszirkel und regelmäßige Mitarbeitergespräche mit Zielvereinbarung.

> die Leitung physiotherapeutische Fachkompetenz und eine große Sozialkompetenz benötigt, zum Beispiel Kommunikations- und Durchsetzungsvermögen, sowie Persönlichkeitskompetenz, etwa die Bereitschaft, Verantwortung zu übernehmen.

\section{Fazit}

Zusammenfassend kann Britta-Julia Kreuzer festhalten, dass ...

$>$ leitende Therapeuten entsprechend weiterqualifiziert werden müssen, um die an sie gestellten Anforderungen zur Mitarbeiterführung ausreichend erfüllen zu können.

$>$ Kliniken das Anforderungsprofil an ihre physiotherapeutische Leitung kritisch reflektieren sollten. Wird beispielsweise ein solch umfassendes BWL-Wissen gefordert, dass dafür ein Studium notwendig ist, wäre eine Möglichkeit, die fachliche und die organisatorische Leitung voneinander zu trennen.

Eva Trompetter

$\Rightarrow$ Kreuzer BJ. Personalmanagement in der stationären Physiotherapie unter besonderer Berücksichtigung der Führungskompetenzen. Bachelorarbeit an der Hochschule Hildesheim; 2010 